\title{
Molecular and functional profiling of human islets: from heterogeneity to human phenotypes
}

\author{
Rafael Arrojo e Drigo ${ }^{1}$ (1) - Birbickram Roy ${ }^{2,3}$ (1) $\cdot$ Patrick E. MacDonald ${ }^{2,3}$ (i)
}

Received: 2 March 2020 / Accepted: 6 April 2020 / Published online: 31 August 2020

(C) Springer-Verlag GmbH Germany, part of Springer Nature 2020

\begin{abstract}
Efforts to phenotype pancreatic islets have contributed tremendously to our present understanding of endocrine function and diabetes. A continued evolution in approaches to study islet physiology is important given the need to establish reference points for mature islet functionality, understanding biological variation amongst individuals and cells, and the ongoing appreciation of the role for islets in diabetes susceptibility. Recent efforts in islet biology have focused on technological improvements in imaging, molecular profiling and data analysis, along with a push for enhanced transparency and reporting. The integration of these approaches within a classical islet physiology framework, and approaches to link these data with in vivo human phenotypes, will be critical as we move towards a better understanding of islet function in health and disease. Here we discuss what we feel are important issues and useful approaches to consider as we move forward as a field in islet and beta cell phenotyping.
\end{abstract}

Keywords Diabetes · Glucagon · Human · Insulin · Islets of Langerhans · Phenotyping, cellular heterogeneity $\cdot$ Review $\cdot$ Single cell

$\begin{array}{ll}\text { Abbreviations } \\ \text { ADI } & \text { Alberta Diabetes Institute } \\ \text { GWAS } & \text { Genome-wide association study } \\ \text { HPAP } & \text { Human Pancreas Analysis Program } \\ \text { IIDP } & \text { Integrated Islet Distribution Program } \\ \text { scRNA-seq } & \text { Single-cell RNA sequencing }\end{array}$

Electronic supplementary material The online version of this article (https://doi.org/10.1007/s00125-020-05159-8) contains a slide of the figure for download, which is available to authorised users.

Patrick E. MacDonald

pmacdonald@ualberta.ca

1 Molecular and Cell Biology Laboratory, Salk Institute for Biological Studies, La Jolla, CA, USA

2 Department of Pharmacology, University of Alberta, Edmonton, AB, Canada

3 Alberta Diabetes Institute, LKS Centre, University of Alberta, Rm. 6-112, Edmonton, AB T6G 2E1, Canada

\section{Introduction}

Through a series of electrical, $\mathrm{Ca}^{2+}$ and molecular signalling processes, endocrine cells within the pancreatic islets of Langerhans regulate the release of key metabolismregulating hormones. Early characterisation of these cells primarily consisted of histopathological analyses and indirect assessment of beta cell activity by measurement of circulating hormones or C-peptide. Modelling extended these measurements to extract indices of islet function [1]. Dynamic measurements in vivo and in isolated perfused pancreases provided key insights into islet function, such as the dynamic properties of insulin release and its $\mathrm{Ca}^{2+}$-dependence, yielding models of biphasic insulin secretion that remain foundational [2]. The establishment of methods to isolate islets, first by microdissection and then by digestion and density centrifugation, ushered in a new era in the study of islets and islet cells that continues today [3].

The key roles for cellular metabolism, excitability and entry of $\mathrm{Ca}^{2+}$ as key determinants of insulin secretion were demonstrated in studies throughout the 1970s to 1990s, which established what we now think of as key islet and beta cell 'functional phenotypes'. In beta cells, these include glucosedependent metabolic activity and resultant signal generation, ATP-dependent action-potential firing, oscillations in 
intracellular $\mathrm{Ca}^{2+}$ and dynamic biphasic insulin secretion [4]. Characteristic alterations in these phenotypes, for example the loss of pulsatile $\mathrm{Ca}^{2+}$ responses and insulin output, are thought to define islet dysfunction in diabetes. Importantly, these functional phenotypes have long been known to vary between individuals, between islets from the same individual and even amongst different beta cells. Although the factors driving heterogeneity at these levels remain to be fully elucidated, it is likely they are determined, at least in part, by the underlying 'molecular phenotypes' of these individuals, islets and cells. Genetic variation can now be linked to insulin secretory responses in individuals [5] and to the secretory responses of isolated islets [6]. Extensive genomic phenotyping of human islets and single cells promises to unravel relevant regulatory and pathophysiological mechanisms, and this has been recently reviewed [7].

At the single-cell level, improved technologies and analytical methods, principally in molecular profiling of transcript and protein expression, have extended our understanding of human beta cell heterogeneity, and advanced methodologies in imaging and network analysis suggest important roles for beta cell subtypes as important contributors to global $\mathrm{Ca}^{2+}$ waves. However, direct links between molecular and functional heterogeneity remain difficult to assess and many of the studies thus far are limited to a relatively modest number of donors. Biological readouts from the same cell also remains rather limited. Phenotyping whole intact islets at scale has proved somewhat easier. Key insights that have been reiterated as a result of newer phenotyping efforts include the substantial variation in insulin secretion amongst islet preparations [8], and important strides have been made in the elucidation of links between metabolic phenotypes and islet genomics [9]. Nonetheless, important challenges remain in understanding the association between the impact of molecular variation on islet function and in vivo glucose homeostasis. Here we discuss human pancreatic islet phenotyping with a focus on improving links between functional and molecular assessment of beta cells and intact islets, and technological approaches that can be used to connect this understanding to in vivo human phenotypes (Fig. 1).

\section{Linking molecular and functional heterogeneity of human beta cells}

Molecular heterogeneity in beta cells Largely due to the emergence of technologies that enable the molecular profiling of single cells on the one hand [10], and functional profiling of interconnected beta cell $\mathrm{Ca}^{2+}$ signalling networks on the other [11], human beta cell heterogeneity has received renewed attention [12]. Most studies examining sufficiently large numbers of beta cells suggest the presence of between three and five molecularly and/or functionally distinct beta cell subpopulations.
Although questions persist about an apparently limited overlap of observed subpopulations and markers between single-cell RNA sequencing (scRNA-seq) studies [13], it remains that this may, in part, be addressed by ongoing efforts to improve the bioinformatic and systems biology approaches used in this context. Recent meta-analyses combining published datasets [14], including our own recent work [15], largely confirm the existence of these beta cell subpopulations across several studies and donors. It seems likely that some of these beta cell subpopulations, in fact, represent 'cellular states' through which the cells cycle at the transcriptional and functional levels $[14,16]$. For example, the cycling of beta cells through states of high stress vs high insulin expression would be consistent with the recent observation of 'extreme' beta cells, postulated to disproportionately contribute to basal insulin secretion [17]. Our analysis suggests three beta cell states consistent with: high levels of beta cell identity and functional markers (state 1); high expression of p53, along with Wnt and insulin-IGF1 signalling (state 2); and ribosomal biogenesis and mRNA translation (state 3) [15].

Functional heterogeneity in beta cells It is often assumed that the different beta cell states translate directly to differential functionality. For example, populations expressing higher levels of the glucose transporter GLUT2 (encoded by $S L C 2 A 2$ ) or GLP-1 receptor (encoded by GLP1R) are thought to be 'more functional'. This is perhaps an over-simplistic interpretation and only a single study, to our knowledge, has successfully linked molecularly distinct human beta cell states (or subpopulations) with the measurement of insulin secretion from beta cell subpopulations sorted through the use of two cell surface markers [18]. While some markers (of stress or dedifferentiation, for example) may indeed delineate 'poorly functional' beta cells, it should be considered that beta cells with low insulin-secretion rates may be important for islet function. This is suggested by recent studies on 'hub' or 'leader' beta cells that represent a beta cell state (which may or may not be stable over time) that exerts disproportionate control of $\mathrm{Ca}^{2+}$ signalling when present in situ $[11,19]$. Hub/leader beta cells have high levels of metabolic enzymes (i.e. glucokinase), increased mitochondrial density and function, and highly responsive $\mathrm{Ca}^{2+}$ signals; yet, they have low expression of insulin and beta cell identity genes [11]. Similarly, we ask: are low-insulin-secreting but proliferative beta cells considered 'non-functional', or are they performing a function that represents important physiology? We may need to re-think 'functionality' within the context of heterogeneous beta cell populations and how this translates to whole-islet physiology and in vivo phenotypes.

Linking molecular and functional heterogeneity One approach to directly link single-cell molecular profiling and functional assessment uses combined or sequential measurement of multiple parameters per cell. One such approach to 
Fig. 1 Functional and molecular phenotyping of human islets from single cells to in vivo human phenotypes. This figure is available as a downloadable slide

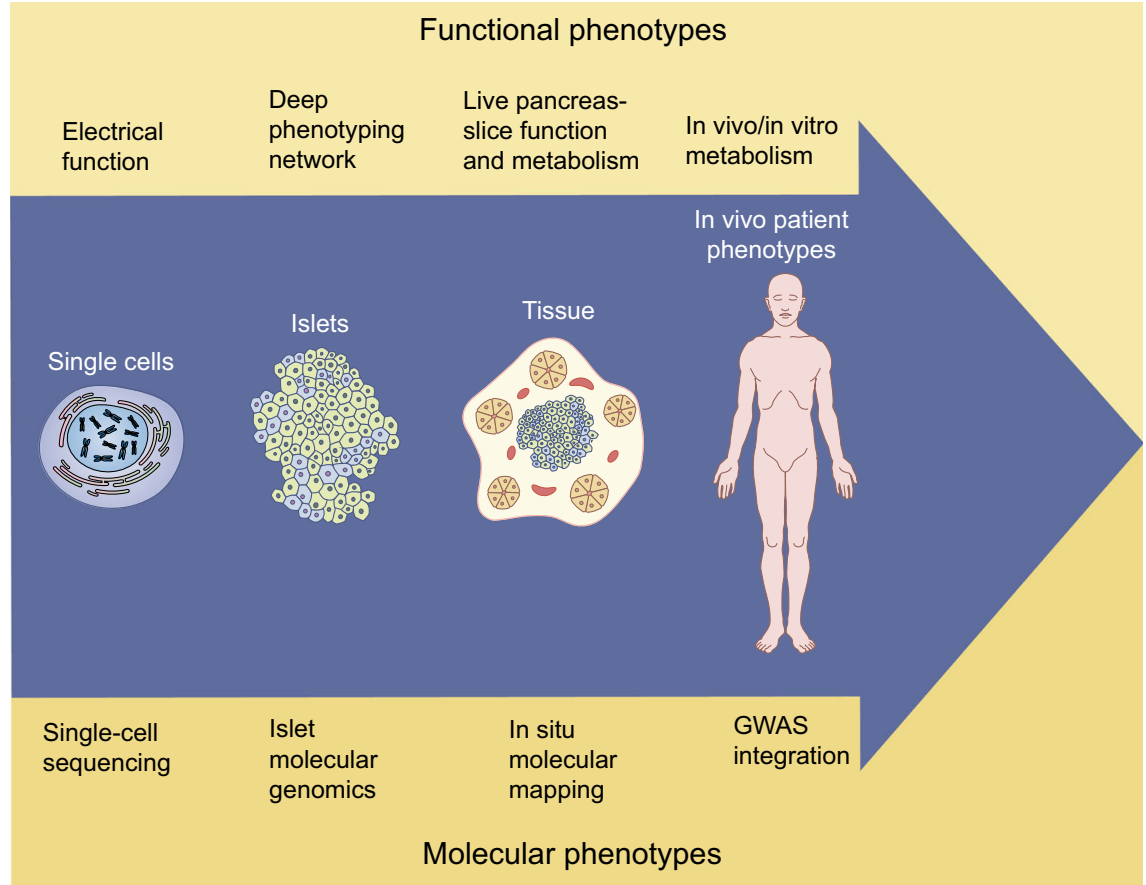

multi-modal profiling links electrophysiological and transcriptomic profiling: a method called Patch-seq [20]. We recently integrated Patch-seq into our human islet isolation and phenotyping programme in Edmonton (www.isletcore. ca) and have, so far, reported the combined function and transcriptional profiles of $>1300$ human islet cells [21]. This provides a resource describing correlations between $\sim 5000$ transcripts and beta cell properties, such as $\mathrm{Ca}^{2+}$ and $\mathrm{Na}^{+}$ channel activities and insulin granule exocytosis, and has provided evidence in support of dysfunction-driving pathways in type 2 diabetes. Intriguingly, we report some strong functional differences amongst beta cell subpopulations using previously reported heterogeneity markers, particularly RBP4 [10], and evidence that some metabolic pathways actually correlate best with 'low-exocytosis' beta cells, perhaps indicating a role for these as a 'hub-like' population that are more directly involved in glucose-sensing and $\mathrm{Ca}^{2+}$ signalling than in insulin exocytosis per se. Because of limitations in throughput with the Patch-seq approach, however, it is difficult to directly look for small hub-like subpopulations in the current dataset. However, approaches in 'predictive electrophysiology' [21] and spatial transcriptomics [17] should eventually allow mapping of functional properties onto larger scRNA-seq datasets, and translation of these in situ.

\section{Seeing is believing: beta cell heterogeneity and a role for ageing?}

Beta cell heterogeneity in space and time The limitations of islet cell scRNA-seq studies have recently been discussed
[22]. Also, the interpretation of stress responses may be challenging since some highly stressed cells could be lost and stress markers in cells that do survive tend to rise early after islet dissociation and then dampen after overnight culture [21]. Therefore, such aspects of beta cell heterogeneity must be validated in situ, namely the expression of molecular markers (e.g. at the level of mRNA transcripts and/or protein markers) and aspects of nutrient metabolism and cell secretory function (e.g. $\mathrm{Ca}^{2+}$ and/or secretory granule dynamics [23]). Interesting in this respect are lineage tracing experiments, which have revealed the presence of alpha cell-derived beta cells located in the periphery of the islet [24] and that beta cells can be derived from other cell types upon extreme beta cell loss [25]. Furthermore, the spectrum of beta cell heterogeneity might change over time and during disease; recent studies have found that a subset of beta cells within ageing islets of mice and humans showed an age-dependent increase in the expression of the IGF1 receptor protein (IGF1R) and that these cells also had a senescent cell signature [26]. In fact, these senescent beta cells, which are mainly characterised by expression of the cell-cycle inhibitor CDKN2A (or p16) and by the display of a senescence-associated secretory phenotype (SASP), may constitute a specific beta cell 'state' that contributes towards the dysfunction and/or death of neighbouring beta cells [27].

Novel imaging technologies to map beta cell heterogeneity Recently, the existence of different types of beta cells, including proliferative beta cells, has been visualised using in situ mass cytometry. Here, cells are labelled with a panel of antibodies conjugated to heavy metals and imaged using time of 
flight (TOF) mass spectrometry, which can achieve single-cell resolution [28]. This approach identified three different clusters (or states) of beta cells based on the expression of cell identity markers (e.g. pancreatic and duodenal homeobox 1 [PDX1], C-peptide), proliferation markers (i.e. ki67) or markers previously associated with beta cell heterogeneity (i.e. CD9 and ST8SIA1) [18]. Perhaps the most welldocumented aspect of beta cell heterogeneity is the presence of rare ki67-positive beta cells, which are thought to be in a proliferative state (or that have at least activated the cell replication programme) and decrease in number with age [25]. Proliferating beta cells have an immature phenotype, not unlike the hub-cell population described above, characterised by an overall low expression of beta cell identity and function genes [26]. Interestingly, ki67-positive beta cells can be found in at least two different subtypes of beta cells in humans, which in turn may change as a function of age [28].

The age of beta cells These observations suggest that most beta cells could remain largely quiescent for long periods of time (some of these may become senescent over time), while rare beta cells can replicate and generate new beta cells in adults [28], and that the relative frequency of different beta cell states could change as a function of age, ultimately suggesting that the makeup of beta cell heterogeneity is dynamic. As a result, the phenotype of beta cells may be in a certain state of molecular and temporal 'flux', ultimately creating a heterogeneous scenario in which beta cells could have changing molecular and/or functional phenotypes over their lifetime, and also vastly different ages. Accordingly, mathematical modelling [29] and direct measurements of ${ }^{14} \mathrm{C}$ in beta cells from humans of different ages suggests that formation of new beta cells occurs in the first three decades of life [30]. Furthermore, using a combination of electron microscopy and multi-isotope mass spectroscopy (called 'MIMS-EM' [multi-isotope imaging mass spectrometry-electron microscopy]), we have mapped the longevity of different islet cell types in situ [31]. While most beta and alpha cells and all delta cells were as old as neurons and did not turnover for an entire lifetime, a subpopulation of alpha and beta cells had indeed undergone turnover and, thus, were responsible for the maintenance of islet mass during adulthood [31]. The commitment of these cell types towards a lifelong post-mitotic phenotype occurred in a heterogeneous fashion before and after weaning, a process that triggers changes in beta cell metabolism and functional maturation mediated by a mammalian target of rapamycin (mTOR) to AMPactivated protein kinase (AMPK) switch [32]. Together, these studies support the idea that most human beta cells are remarkably long-lived and, as such, they face a lifetime pressure (which in humans can last for over a century) to maintain their cellular identity and function in order to sustain normal glucose homeostasis. Understanding the different aspects of the beta cell lifetime, and the molecular and functional changes that these cells undergo during the adult human lifetime, will be critical to understanding the basic biology of islets and to develop concepts that could be applied to target studies aimed at preventing beta cell loss or stimulating beta cell regeneration.

\section{Increasing scale: connecting molecular and functional phenotypes in human islets}

Human islet isolation and phenotyping initiatives Studies on beta cell heterogeneity have been performed, so far, on modest numbers of donors due to the technical demands and costs of single-cell profiling, and to the complexity in access to human islets. As the costs of molecular profiling drop, single-cell phenotyping will increasingly be integrated within largescale human islet phenotyping programmes. These initiatives often focus on functional and quality assessment and are propelled, in part, by recent calls for improved human islet reporting [33]. The Integrated Islet Distribution Program (IIDP) Human Islet Phenotyping Program (HIPP) provides functional, viability and basic cell-composition phenotyping of all human islet isolations coordinated by the IIDP and the Alberta Diabetes Institute (ADI) IsletCore [34]. A web tool from our own human islet isolation programme (ADI IsletCore) was recently released (www.isletcore.ca, accessed 1 April 2020) and provides publicly available insulin secretion and quality control data on human islet preparations (now standing at 360 donors) in real time, with gene expression panels available for a subset of these. Other initiatives focus primarily on the molecular phenotyping of human islets, such as the Integrated Network for Systematic analysis of Pancreatic Islet RNA Expression (InsPIRE) group [35]. These reveal important insights into islet gene regulation (as previously reviewed [7]) and make important contributions to understanding the impact of islet-localised genetic signals on in vivo glycaemic traits [9], but no programme has yet released data on the combined molecular and functional profiling of a large number of human islet preparations. 'Islet Gene View', developed by the Excellence of Diabetes (EXODIAB) research group in Sweden and described in a recent preprint [6], may fill this gap once it is made publicly available, by connecting genetic variation, transcript expression and insulin responses in samples from 188 donors.

Expanding phenotyping within networks A limitation of the above initiatives is the narrow phenotyping that occurs beyond molecular genomic profiling. It can be argued that measurement of insulin secretion, even combined with some basic cell-type composition analyses, is a shallow assessment of islet physiology. Ideally, human islet phenotyping programmes will include detailed information on islet function (i.e. metabolic and electrical activity), in situ interactions (i.e. morphology and interaction of beta cells with nerves and blood vessels) and detailed single-cell profiling (i.e. analysis 
of heterogeneity markers). Integrating this kind of deep phenotyping with detailed genomic profiling, genome-wide association study (GWAS) data and in vivo human phenotypes promises to shed important light on the observed variation in islet function, and the contribution of this to the heterogeneity of metabolic phenotypes and type 2 diabetes risk. Important strides are indeed being made in this direction. The Human Pancreas Analysis Program (HPAP; hpap. pmacs.upenn.edu, accessed 1 April 2020), part of the National Institutes of Health (NIH)-funded Human Islet Research Network (hirnetwork.org, accessed 1 April 2020), focuses on the phenotyping of pancreas from donors with type 1 diabetes and matched control donors [36]. Data from 50 donors are available and the programme has been extended to include samples from donors with type 2 diabetes. This effort brings together morphological, physiological and genomic analyses with profiling approaches, such as imaging mass cytometry, that can assess beta cell heterogeneity [37]. The focus of this programme on comparing pancreases from individuals with type 1 diabetes and type 2 diabetes with appropriately matched control donors will provide valuable data for the study of disease pathogenesis, and the provision of data openly and in 'real time' should be applauded. However, the ability to integrate the deep physiological phenotyping on very large numbers of preparations required for insight into human functional variation remains somewhat unclear and challenges in dataset integration remain.

Moving towards in vivo human phenotypes Data from HPAP may eventually be used to link detailed islet phenotypes to in vivo human metabolic function by integration with GWAS and phenome-wide association studies (PheWAS), as has been done with islet transcript-expression data [9]. More direct connections between islet molecular and physiological profiles can, however, be facilitated by the study of tissues taken directly from metabolically phenotyped individuals. As part of the Innovative Medicines Initiative in Diabetes (IMIDIA) programme, the Risk Assessment and ProgreSsiOn of Diabetes (RHAPSODY) Consortium have been collecting pancreas samples from metabolically phenotyped patients undergoing partial pancreas resection [38]. Notwithstanding the potential impacts of the pancreatic tumours being resected on metabolic phenotypes, this approach has allowed the direct linking of islet transcript profiles and expression quantitative trait loci (eQTL) with impaired glucose tolerance and type 2 diabetes in patients [39] and may address some of the quality concerns inherent to the study of islets isolated from pancreases of organ donors, such as the potential impact of prolonged ischaemic times or hospital stays [38]. This approach is now suggested as a source of living donor tissue and live-islet assessment [40] which would allow direct correlations spanning from cell physiology, to molecular genetic and genomic profiling, to in vivo human phenotypes. Although many patient samples (>100) have been assessed for molecular profiling using lasercaptured islets from fixed pancreas samples, it remains to be determined how efficiently this can be adapted to the in vitro assessment of live islets. Importantly, a very recent study [41] has now successfully demonstrated the in vitro islet dysfunction within live pancreas slices from individuals with impaired glucose tolerance or type 2 diabetes.

\section{Perspective}

The development of modern high-throughput sequencing platforms has injected a fresh perspective into the idea that beta cells are indeed heterogeneous, first observed nearly three decades ago [42, 43]. Importantly, the integration of such methods with high-resolution functional, spatiotemporal and molecular phenotyping, and microscopy pipelines has the potential to provide additional insights and allow the dissection of pathways involved in the heterogeneity of, not only beta cells, but also other endocrine and exocrine cell types,

\section{Connecting molecular and functional profiles of human islets: from cells to tissues to people}

- Pancreatic beta cells, heterogeneous in their molecular and secretory profiles, work together within intact pancreatic islets to control insulin output and glucose homeostasis.

- Insulin secretory function, in itself, varies amongst individuals, in part due to diverse genetic and environmental influences.

- Multi-modal single-cell approaches, such as pancreas Patch-seq, can link molecular and functional profiling in individual beta cells.

- Mapping of functional markers within intact tissue and pancreas slices can help reveal spatial interactions and phenotypes.

- Continued integration of data from human islet isolation and phenotyping networks can link genetic variation and signals for diabetes risk with islet function.

- A move towards linked patient profiling and pancreas/islet phenotyping will improve our understanding of the links between islet biology and metabolic phenotypes. 
and the relevance of this heterogeneity to cell function and overall human glucose homeostasis in health and disease.

Acknowledgements We thank the Trillium Gift of Life Network (TGLN) and Human Organ Procurement and Exchange (HOPE) programs in Canada for their work in the identification and procurement of human pancreas, and organ donors and their families for their generous gifts to research over many years.

Funding Work in the authors laboratories is funded by grants from the National Institutes of Health Research - Human Islet Research Network (RAeD and PEM), the JDRF (PEM), the American Diabetes Association (RAeD) and a Foundation Grant from the Canadian Institutes of Health Research (PEM). PEM's group is also contributing to the NIH HPAPT2D pilot initiative.

Authors' relationships and activities The authors declare that there are no relationships or activities that might bias, or be perceived to bias, their work.

Contribution statement RAeD, BR and PEM each wrote and revised the manuscript. All authors approved the version to be published.

\section{References}

1. Matthews DR, Hosker JP, Rudenski AS, Naylor BA, Treacher DF, Turner RC (1985) Homeostasis model assessment: insulin resistance and $\beta$-cell function from fasting plasma glucose and insulin concentrations in man. Diabetologia 28(7):412-419. https://doi. org/10.1007/BF00280883

2. Grodsky GM (1972) A threshold distribution hypothesis for packet storage of insulin and its mathematical modeling. J Clin Invest 51(8):2047-2059. https://doi.org/10.1172/JCI107011

3. Piemonti L, Pileggi A (2013) 25 years of the Ricordi automated method for islet isolation. CellR4 Repair Replacement Regen Reprogramming 1(1):e128

4. MacDonald PE, Joseph JW, Rorsman P (2005) Glucose-sensing mechanisms in pancreatic beta-cells. Philos Trans R Soc Lond Ser B Biol Sci 360(1464):2211-2225. https://doi.org/10.1098/rstb. 2005.1762

5. Wood AR, Jonsson A, Jackson AU et al (2017) A genome-wide association study of IVGTT-based measures of first-phase insulin secretion refines the underlying physiology of type 2 diabetes variants. Diabetes 66(8):2296-2309. https://doi.org/10.2337/db161452

6. Asplund O, Storm P, Ottosson-Laakso E et al (2019) Islet Gene View - a tool to facilitate islet research. Biorxiv 435743 (Preprint). 28 March 2019. Available from: https://doi.org/10. 1101/435743. Accessed: 1 April 2020

7. Mattis KK, Gloyn AL (2020) From genetic association to molecular mechanisms for islet-cell dysfunction in type 2 diabetes. J Mol Biol 32(5):1551-1578

8. Kayton NS, Poffenberger G, Henske J et al (2015) Human islet preparations distributed for research exhibit a variety of insulinsecretory profiles. Am J Phys Endocrinol Metab 308(7):E592E602. https://doi.org/10.1152/ajpendo.00437.2014

9. Ng NHJ, Willems SM, Fernandez J et al (2019) Tissue-specific alteration of metabolic pathways influences glycemic regulation. Biorxiv 790618 (Preprint). 3 October 2019. Available from: https://doi.org/10.1101/790618. Accessed: 1 February 2020
10. Segerstolpe $\AA$, Palasantza A, Eliasson P et al (2016) Single-cell transcriptome profiling of human pancreatic islets in health and type 2 diabetes. Cell Metab 24(4):593-607. https://doi.org/10.1016/j. cmet.2016.08.020

11. Johnston NR, Mitchell RK, Haythorne E et al (2016) Beta cell hubs dictate pancreatic islet responses to glucose. Cell Metab 24(3):389 401. https://doi.org/10.1016/j.cmet.2016.06.020

12. Benninger RKP, Hodson DJ (2018) New understanding of $\beta$-cell heterogeneity and in situ islet function. Diabetes 67(4):537-547. https://doi.org/10.2337/dbi17-0040

13. Wang YJ, Kaestner KH (2019) Single-cell RNA-Seq of the pancreatic islets - a promise not yet fulfilled? Cell Metab 29(3):539-544. https://doi.org/10.1016/j.cmet.2018.11.016

14. Xin Y, Gutierrez GD, Okamoto $\mathrm{H}$ et al (2018) Pseudotime ordering of single human $\beta$-cells reveals states of insulin production and unfolded protein response. Diabetes 67(9):1783-1794. https://doi. org $/ 10.2337 / \mathrm{db} 18-0365$

15. Arrojo e Drigo R, Erikson G, Tyagi S et al (2019) Aging of human endocrine pancreatic cell types is heterogeneous and sex-specific. Biorxiv 729541 (Preprint). 8 August 2019. Available from: https:// doi.org/10.1101/729541. Accessed: 1 February 2020

16. Modi H, Skovsø S, Ellis $\mathrm{C}$ et al (2019) Ins2 gene bursting activity defines a mature $\beta$-cell state. Biorxiv 702589 (Preprint). 14 July 2019. Available from: https://doi.org/10.1101/702589. Accessed: 1 February 2020

17. Farack L, Golan M, Egozi A et al (2018) Transcriptional heterogeneity of beta cells in the intact pancreas. Dev Cell 48(1):115-125

18. Dorrell C, Schug J, Canaday PS et al (2016) Human islets contain four distinct subtypes of $\beta$ cells. Nat Commun 7(1):11756. https:// doi.org/10.1038/ncomms 11756

19. Salem V, Silva LD, Suba K et al (2019) Leader $\beta$-cells coordinate $\mathrm{Ca}^{2+}$ dynamics across pancreatic islets in vivo. Nat Metabolism 1(6):615-629. https://doi.org/10.1038/s42255-019-0075-2

20. Cadwell CR, Palasantza A, Jiang X et al (2016) Electrophysiological, transcriptomic and morphologic profiling of single neurons using patch-seq. Nat Biotechnol 34(2):199 203. https://doi.org/10.1038/nbt.3445

21. Camunas-Soler J, Dai X, Hang Y et al (2020) Pancreas patch-seq links physiologic dysfunction in diabetes to single-cell transcriptomic phenotypes. Cell Metab. https://doi.org/10.1016/j. cmet.2020.04.005

22. Mawla AM, Huising MO (2019) Navigating the depths and avoiding the shallows of pancreatic islet cell transcriptomes. Diabetes 68(7):1380-1393. https://doi.org/10.2337/dbi18-0019

23. Gandasi NR, Yin P, Omar-Hmeadi M, Laakso EO, Vikman P, Barg S (2018) Glucose-dependent granule docking limits insulin secretion and is decreased in human type 2 diabetes. Cell Metab 27(2): 470-478. https://doi.org/10.1016/j.cmet.2017.12.017

24. van der Meulen T, Mawla AM, DiGruccio MR et al (2017) Virgin beta cells persist throughout life at a neogenic niche within pancreatic islets. Cell Metab 25(4):911-926. https://doi.org/10.1016/j. cmet.2017.03.017

25. Thorel F, Népote V, Avril I et al (2010) Conversion of adult pancreatic alpha-cells to beta-cells after extreme beta-cell loss. Nature 464(7292):1149-1154. https://doi.org/10.1038/nature08894

26. Aguayo-Mazzucato C, van Haaren M, Mruk M et al (2017) $\beta$-Cell aging markers have heterogeneous distribution and are induced by insulin resistance. Cell Metab 25(4):898-910. https://doi.org/10. 1016/j.cmet.2017.03.015

27. Thompson PJ, Shah A, Ntranos V, Gool FV, Atkinson M, Bhushan A (2019) Targeted elimination of senescent beta cells prevents type 1 diabetes. Cell Metab 29(5):1045-1060. https://doi.org/10.1016/j. cmet.2019.01.021

28. Wang YJ, Golson ML, Schug J et al (2016) Single-cell mass cytometry analysis of the human endocrine pancreas. Cell Metab 24(4): 616-626. https://doi.org/10.1016/j.cmet.2016.09.007 
29. Cnop M, Hughes SJ, Igoillo-Esteve M et al (2009) The long lifespan and low turnover of human islet beta cells estimated by mathematical modelling of lipofuscin accumulation. Diabetologia 53(2):321-330. https://doi.org/10.1007/s00125-009-1562-x

30. Gregg BE, Moore PC, Demozay D et al (2012) Formation of a human $\beta$-cell population within pancreatic islets is set early in life. J Clin Endocrinol Metab 97(9):3197-3206. https://doi.org/10.1210/ jc.2012-1206

31. Arrojo E, Drigo R, Lev-Ram V, Tyagi S et al (2019) Age mosaicism across multiple scales in adult tissues. Cell Metab 30(2):343-351. https://doi.org/10.1016/j.cmet.2019.05.010

32. Jaafar R, Tran S, Shah A et al (2019) mTORC1 to AMPK switching underlies $\beta$-cell metabolic plasticity during maturation and diabetes. J Clin Investig 130:4124-4137

33. Hart NJ, Powers AC (2018) Use of human islets to understand islet biology and diabetes: progress, challenges and suggestions. Diabetologia 62(2):212-222. https://doi.org/10.1007/s00125-0184772-2

34. Brissova M, Niland JC, Cravens J, Olack B, Sowinski J, EvansMolina C (2019) The integrated islet distribution program answers the call for improved human islet phenotyping and reporting of human islet characteristics in research articles. Diabetologia 62(7): 1312-1314. https://doi.org/10.1007/s00125-019-4876-3

35. Viñuela A, Varshney A, Bunt M van de et al (2019) Influence of genetic variants on gene expression in human pancreatic islets implications for type 2 diabetes. Biorxiv 655670 (Preprint). 31 May 2019. Available from: https://doi.org/10.1101/655670. Accessed: 1 February 2020

36. Kaestner KH, Powers AC, Naji A, Consortium H, Atkinson MA (2019) NIH initiative to improve understanding of the pancreas, islet, and autoimmunity in type 1 diabetes: The Human Pancreas Analysis Program (HPAP). Diabetes 68(7):1394-1402. https://doi. org/10.2337/db19-0058
37. Wang YJ, Traum D, Schug J et al (2019) Multiplexed in situ imaging mass cytometry analysis of the human endocrine pancreas and immune system in type 1 diabetes. Cell Metab 29(3):769-783. https://doi.org/10.1016/j.cmet.2019.01.003

38. Solimena M, Schulte AM, Marselli L et al (2017) Systems biology of the IMIDIA biobank from organ donors and pancreatectomised patients defines a novel transcriptomic signature of islets from individuals with type 2 diabetes. Diabetologia 61(3):641-657. https:// doi.org/10.1007/s00125-017-4500-3

39. Khamis A, Canouil M, Siddiq A et al (2019) Laser capture microdissection of human pancreatic islets reveals novel eQTLs associated with type 2 diabetes. Mol Metab 24:98-107. https://doi.org/10. 1016/j.molmet.2019.03.004

40. Barovic M, Distler M, Schöniger E et al (2019) Metabolically phenotyped pancreatectomized patients as living donors for the study of islets in health and diabetes. Mol Metab 27S:S1-S6. https://doi.org/10.1016/j.molmet.2019.06.006

41. Cohrs CM, Panzer JK, Drotar DM et al (2020) Dysfunction of persisting $\beta$ cells is a key feature of early type 2 diabetes pathogenesis. Cell Rep 31(1):107469. https://doi.org/10.1016/j.celrep.2020. 03.033

42. Kiekens R, 't Veld PI, Mahler T, Schuit F, Winkel MVD, Pipeleers D (1992) Differences in glucose recognition by individual rat pancreatic B cells are associated with intercellular differences in glucose-induced biosynthetic activity. J Clin Invest 89(1):117125. https://doi.org/10.1172/JCI115551

43. Pralong WF, Bartley C, Wollheim CB (1990) Single islet beta-cell stimulation by nutrients: relationship between pyridine nucleotides, cytosolic $\mathrm{Ca}^{2+}$ and secretion. EMBO J 9(1):53-60. https://doi.org/ 10.1002/j.1460-2075.1990.tb08079.x

Publisher's note Springer Nature remains neutral with regard to jurisdictional claims in published maps and institutional affiliations. 